\title{
Nursing Care of the Family with a Chronically III, Hospitalized Child: An Alternative Approach
}

Adelina R. Ferraro, R.N., M.S.N. Diane C. Longo, R.N., M.N.

\begin{abstract}
Although nurses successfully employ family crisis intervention strategies when caring for pediatric in-patients, this approach may be counterproductive with families of a chronically ill, hospitalized child. During an admission to an acute care facility these families, if previous levels of functioning have been adequate, may be able to function well despite the stress that all families experience under such circumstances. This article explores an alternative framework for assessing families who are coping simultaneously with a chronic pediatric condition and an acute medical problem. An assessment model based on Miller's (1983) work was developed; it focuses on families' response to long-term health problems and successful adaptation. A clinical case is presented and analyzed using relevant theoretical and research literature. Nursing interventions with both family and staff are included in this presentation.
\end{abstract}

Hospitalization of a child can precipitate significant stress for both patient and family, which can in turn result in family crisis. Should a crisis state continue to escalate without intervention, eventual breakdown in family functioning can occur. Fortunately, as Williams (1978) suggests, through utilization of a crisis intervention framework-which includes such activities as (a) assuring a realistic

Adelina R. Ferraro, R.N., M.S.N. (Rho), is Staff Nurse-Clinical Research Unit, Children's Hospital, Boston.

Dianne C. Longo, R.N., M.N. (Rho), is Assistant Professor of Parent Child Nursing, The University of Michigan, Ann Arbor.

Summer, 1985, Volume XVII, No. 3 view of the situation, (b) exploring and supporting coping skills, and (c) expanding support networksnurses can assist families in crisis toward resolution and adaptation before dysfunction arises.

However, hospitalization does not precipitate a state of crisis for all children and their families. In addition, clinical interventions that alleviate the stressors experienced by some families may unnecessarily tax the psychosocial well-being of others. For example, while providing simple, repetitive, and concrete medical explanations to the family in crisis helps them process and absorb important information, using this approach with families that are capable of making judgments at a more abstract level may impede rather than promote collaboration and decision making.

Nurses often intervene with families of chronically ill, hospitalized children under the assumption that they are in a state of crisis. In addition, as Longo and Bond (1984) point out, many nurses and other health care professionals assume that dysfunction is a normative pattern in families with chronically ill or disabled children. Clinicians then expect to encounter problems in family functioning where none may exist. When this situation occurs, inappropriate and counterproductive interventions may result.

The purpose of this paper is to explore an alternative framework for assisting families that are coping simultaneously with a chronic, disabling pediatric condition and an acute medical problem. A family seen by the pri- mary author is first presented and is followed by clinical data that are analyzed from the perspective of coping and adaptation rather than of crisis theory. A model for assessment and intervention based on Miller's (1983) work is then reviewed. The article concludes with nursing strategies and outcome descriptions.

\section{The Family}

Michael C. was a seven-year-old, severely retarded, epileptic child who had been healthy until he sustained serious head injuries as a result of a car accident at the age of five years. He was one of seven children (ages 15 months to 15 years). His father was a construction worker; his mother, a homemaker.

Developmentally, Michael functioned at the level of an infant; he could not sit, crawl, walk, or talk. He did, however, respond to his environment, especially maternal interaction or the lack thereof. For example, his episodes of crying and posturing diminished significantly whenever his mother held him or talked to him.

In March of 1983 Michael C. was admitted to a pediatric unit with probable aspiration pneumonia. Six weeks prior to admission he had undergone tendon release surgery, which resulted in a marked decrease in appetite and significant weight loss (weight < 5th percentile). His immediate medical problems included (a) compromised respiratory status secondary to pneumonia, (b) cachetic metabolic state, and (c) increased seizure activity. Medical management during Mi- 
Nursing Care

chael's hospitalization consisted of IV antibiotic therapy, short-term peripheral hyperalimentation, intermittent placement of an NG tube for feedings, readjustment of seizure medications, and eventual surgery for GT tube placement, which was followed by yet another bout of pneumonia requiring additional antibiotics.

Not more than three days after Michael's admission to the unit, considerable tension had arisen between the family and the staff members. The nursing staff perceived the family as being unrealistic, inappropriate, and even uncooperative at times. The family in turn perceived the staff as being unsupportive, restrictive, and domineering. At this point the nursing staff referred the family to the primary writer for clinical assessment and intervention.

Based on the severe nature of the child's chronic disability, previous critical care experience with families in crisis, and the staff's perceptions, a tentative assessment of family crisis and family dysfunction was made. Several problems were thus anticipated: inability of the parents to realistically appraise their son's medical problems, diminished coping skills, and insufficient support networks. It was further expected that long-standing maladaptive responses might be uncovered such as maternal guilt over the child's condition, sibling dysfunction, and marital instability.

After spending one afternoon with Michael and his parents it became clear that there was little evidence to support the initial assessment of crisis and family dysfunction. Despite the fact that this child required an elaborate array of nursing interventions, the family had been providing that care at home on a daily basis and were completely at ease caring for him. Their coping skills appeared more than adequate. Rather than having an unrealistic appraisal of their son's situation, Mr. and Mrs. C were knowledgeable about Michael's past and present medical condition. Not one, but many support networks were at their disposal including neighbors, respite care workers, a parent support group for multihandicapped children, and a
Christian fellowship group.

In discussion with the parents about the family as a unit, neither parent reported problems with siblings, and both described their deliberate attempts to attend to their other children's needs. In addition, the mother refuted the notion of inappropriate and extensive maternal guilt with such statements as "These things just happen, it was no one's fault." The marital dyad also had "weathered the initial storm" of the accident, indeed had grown stronger in the process.

It was concluded that the crisis intervention framework on which nursing care had been based for this family was inappropriate and seemingly counterproductive. Consequently other frameworks to explain the family's response to their child's medical problems and hospitalizations were sought. One afternative framework for assisting families with a chronically ill, hospitalized child is the "Family Power Resource Model." This framework is based on the "Patient Power Resources" model set forth by Miller (1983). See Figure 1.

\section{The Family Power Resources Model}

Miller (1983) defined power as "the ability to influence what happens to oneself." In her Patient Power Re- sources model, power is further defined as nutrient or nuturtant power: "providing for and caring for self; directing others regarding self-care and being the ultimate decision maker regarding care" (p. 3).

Expanding on Miller's concepts, family power is defined as the ability to influence what happens to one's family. Family nurturant power in turn describes the ability to provide for and care for the family, to direct others regarding the care of the family, and to be the ultimate decision maker regarding the care of the family. The ability to effect change in one's family care or to prevent such change is thus the ultimate goal of the Family Power Resources model.

Power is a crucial issue for any family with a hospitalized child; this is especially true for the family of a hospitalized, chronically ill child. The unique features of chronic illness force parents to become knowledgeable and skillful in areas of child care that are often the domain of professionals. The imposing nature of treatment protocols substantially shade patterns of daily living, and the family learns to incorporate the child into the family system with as little disruption as possible. If the family is to adapt in a positive manner, changes in roles, task distribution, and financial priorities must

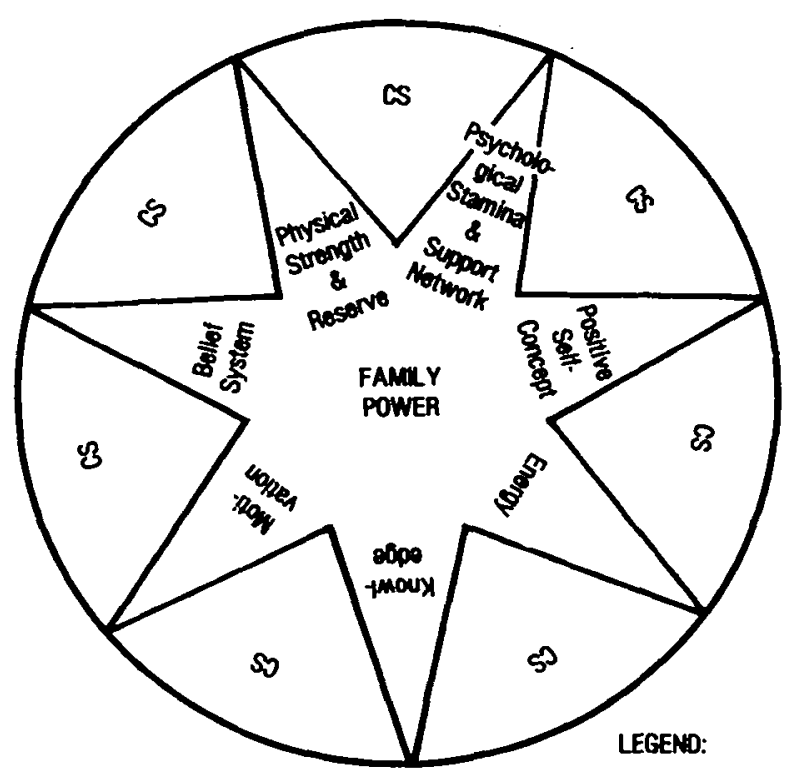

Figure 1. Pationt power resources model.

$C S=$ Coping strategies

Sounce: Miler, J. F. (1983). Patient Power Resources. In J. F. Miller (Ed.), Coping with Chronic illness: Overcoming powerlessness; F. A. Davis, Philadelphia. 
all occur (Anderson, 1981; Pinyerd 1983; Reif, 1975).

When treatment protocols during intermittent hospitalizations of a chronically ill child fail to take into account previous and subsequent home management, continued family adaptation may be threatened. Certain treatments and procedures, though of merit in the hospital setting, can be extremely difficult to maintain within the home setting. To minimize the disruptive impact that a treatment protocol may have on total family functioning, parents must be included in the decision-making process whenever possible. In other words, parental control needs to be maintained, and family powerlessness should be avoided at all costs. The Family Power Resources model provides such a framework for facilitating continued nurturant power for the family with a chronically ill, hospitalized child. This model will be examined in detail.

According to the Family Power Resources model, family power (and thus coping strategies) can be enhanced during hospitalization through the support of one or more of the family's power resources (see Fig. 1): physical strength and reserve, psychological stamina and support network, positive self-concept, energy, knowledge, motivation, and belief system. As can be seen in the discussion of these resources below, use of these concepts is also supported in the literature by authors who concern themselves with healthy coping and adaptation in families with chronically ill children.

Physical Strength and Reserve

Physical strength and reserve refers to the family's current physical functioning and available emergency stores on which to draw in times of stress. Total family physical strength and reserve, moreover, is determined by four interdependent factors: (a) the physiological functioning of each family member, (b) the strength of the marital dyad, (c) the strength of sibling support, and (d) the health of the family system itself. When one or more of these interdependent factors is less than optimal, overall family strength diminishes. As White (1974) found in his research on coping and adaptation, when the internal organization of the system gets "too far off balance" adaptive behavior is seriously hampered.

\section{Psychological Stamina and Support Networks}

Psychological stamina and support networks involve the resiliency of both individual family members and of the system as a whole. Psychological strength rather than physical strength is the focus here. The continuing stressors of chronic illness can wear down the armor of even the strongest of families, and efforts to maintain psychological resiliency is determined in large part by one's available support networks. Resiliency is especially crucial for the family with a chronically ill child. Research has demonstrated that greater adaptation is found among those families who share the burden of their children's illness, both within and outside the family system. More specifically, when individual family members divide the care-giving tasks among themselves, greater adaptation is evident (McKeever, 1981; Tropauer, Franz \& Dilgard, 1970; Ventner, 1981). In such families, siblings and fathers take on greater household responsibilities and their specific role within the family often expands during the adaptation process (Anderson, 1981; Mattsson \& Gross, 1966; Tropauer, Franz, \& Dilgard, 1970).

Maximizing support systems outside the family is associated with greater adaptation as well. Two specific support systems have been cited in the literature as a source of strength for these families: (a) significant others within the community (Adams \& Lindemann, 1974), and (b) parent groups with children suffering from a similar affliction (Mattsson \& Gross, 1966). A third support system, adequate social services (e.g., respite care, schooling for the physically and/ or mentally impaired), is also of import to such families.

\section{Positive Self-Concepts}

Positive self-concepts of both the parents and the afflicted child need to be reevaluated when chronic illness is diagnosed. Just as parents of a malformed infant need time to grieve over the loss of their "perfect infant" (Solnit \& Stark, 1961; Waechter, 1977 , so parents of the chronically ill child must grieve over the loss of their "healthy child." Over time, the process of normalization seems to occur in families with successful adaptation. As Anderson (1981) points out:

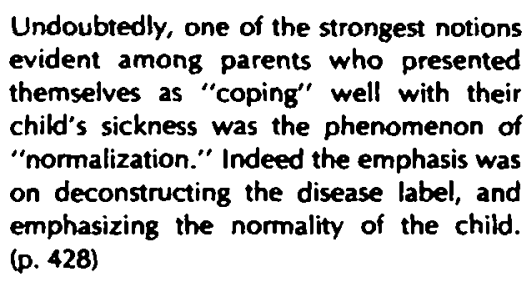

Undoubtedly, one of the strongest notions evident among parents who presented themselves as "coping" well with their child's sickness was the phenomenon of "normalization." Indeed the emphasis was on deconstructing the disease label, and emphasizing the normality of the child. (p. 428)

Although not necessarily labeled as "normalization," this same adaptive technique has been described in four other studies (Adams \& Lindemann, 1974; Kupst et al., 1982; Mattsson \& Gross, 1966; McKeever, 1981). When all family members change in response to the needs of the affected child, the process of normalization is enhanced (Anderson, 1981; Mattsson \& Gross, 1966). When health professionals mislabel this normalization process as denial, family power resources diminish.

\section{Energy}

Energy, the capacity to do work, is utilized to heal physical states, to meet the demands of daily living, to grow, or to cope with unusual stress. Chronic illness often substantially increases the need for energy expenditure among all family members. For example, diseases such as cystic fibrosis, with its time-consuming daily treatments, places high energy demands on the family. Unless adequate nutrition, rest, motivation, and equitable distribution of labor is available, exhaustion may set in. Nursing actions to sustain family energy during hospitalization can do much to promote family power.

\section{Knowledge}

Knowledge is essential for effective decision making, thus avoiding powerlessness. When chronic illness is first diagnosed, Williams (1978) believes that parents need to obtain a "realistic view of the situation" to move in a positive direction. White (1974) adds that long-term coping and adaptation 
require the continuation of this process. Anderson (1981) reports that between $70 \%$ and $90 \%$ of all illness episodes in families with a chronically ill child are handled outside of the formal health care system. Thus parents usually are well-versed in making management decisions for their child. It is not surprising then that discord between staff and family members arises during hospitalization when families are kept in the dark about their child's health status and contemplated treatments.

In her work with fathers of a chronically ill child, McKeever (1981) found that the majority of these fathers were dissatisfied with the factual knowledge they had received regarding the progression of their child's disease. Families need to be kept informed of the disease process, the changing treatment protocols, the options for alternative treatment protocols, and the community resources available to them. Breakdowns in communications are often fueled in part by the many and often changing members of the medical team, particularly in teaching hospitals. If parents want to receive the most up-to-date care for their child, however, teaching hospitals are not easily avoided. Nursing must focus on ensuring adequate knowledge for these families.

\section{Motivation}

Motivation in chronic illness is important in maximizing potential, Miller (1983) stresses, in overcoming the inevitable loss of former social and work roles, and in developing positive self-esteem through risk taking. Ventner (1981) claims that families of a chronically ill child often find that focusing on the present helps them to continue struggling with their daily burdens. The term "belief system" refers to a strong spiritual orientation and/or a philosophical perspective that provides support and comfort during even the most difficult of times. For example, Ventner found that parents who were able to make sense philosophically out of what had happened to them, who were able to "endow the illness with meaning," were better able to cope with a child with cystic fibrosis than those who had not

completed this cognitive/emotional task.

In summary then, using the same framework as Miller (1983) did for the promotion of her Patient Power Resources model, her same argument is made here for the promotion of a Family Power Resource Model:

\begin{abstract}
Families who have a chronically ill child need to have power to be managers of their child's care. They should not forfeit this role to health-care personnel. Nurses can maximize families' resources for pow. er. Nurses help families with deficits in one or more of the power resources by developing remaining intact power resources, as well as by supporting the family's select coping strategies. (p. 11)
\end{abstract}

\section{Clinical Application Of The} Family Power Resource Model

Recall that not more than three days after the admission of profoundly retarded and paralyzed Michael $C$, significant discord between the $C$ family and the staff had arisen. Crisis, although suspected at first, was not the problem; family powerlessness was. Miller (1983) addressed the feeling of powerlessness:

Powerlessness is the perception of the individual that one's actions will not significantly affect an outcome. Powerlessness is a perceived lack of control over a current situation or immediate happening. When one or more of the power resources of physical strength, psychological stamina, self-concept energy, knowledge, motivation and belief system are compromised, powerlessness is a potential problem. (p. 38)

Evidence of the $C$ family's struggle to regain power during those early days of Michael's hospitalization included:

1. Mother's unending request to continue feeding Michael orally (later found to be sustained by her desire to maintain some "normalcy") despite the staff's reluctancy and belief that she was being unrealistic.

2. Mother's frequent removal of $\mathrm{Mi}$ chael from the oxygen tent because "he always looks this way" (i.e., cyanotic) and she felt he needed the physical contact so that he might relax.

3. The parent's refusal to use an NG tube for feeding after discharge no matter what the staff advised (the
NG tube severely agitated Michael and made interacting with him very difficult).

4. The parent's persistant demands for accurate and complete information from the vast medical team.

5. The staff's overall frustration with the frequent demands and independent behaviors exhibited by the family.

It thus appears that the family's power resources of positive self-concept, physical strength and reserve, energy, and knowledge were being compromised during this hospitalization.

To reverse the role of the staff members from compromising power resources to facilitating power resources, their initial diagnosis of crisis and dysfunction within the $C$ family had to be abandoned. No longer could they operate under their previous assumptions. This crisis/dysfunction label was dismantled using the following interventions:

1. Charting Mrs. C's realistic appraisal of Michael's nutritional needs and her interpretation of the advantages and disadvantages of the various feeding plans available.

2. Engaging in informal discussions with the head nurse, assistant head nurse, primary nurse, and social worker about the many social services and social supports currently used by this family.

3. Demonstrating to the staff the extremely potent and positive effect that Mrs. C had upon her son, while still maintaining her role as mother to the other children.

In this way the staff came to view parental behaviors in a more positive light.

Specific strategies to restore those family power resources that had been compromised were implemented. As can be seen in Table 1 the family's self-concept was promoted through recognition of their vast coping skills to date and through support of the mother's desire to maintain the normalcy of Michael's feeding. The need for additional supplements was acknowledged by the mother and permission to insert a GT tube was granted. In this way both the family and the medical staff felt comfortable with the 
TAEE 1

RESTOUnG THE G FALU Y'S POWEA RESOUnCES

\begin{tabular}{|c|c|}
\hline Powne Rescimes & intermations \\
\hline 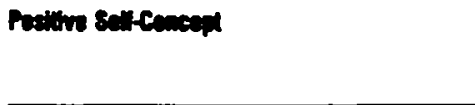 & $\begin{array}{l}\text { Recognize past and present coping stills } \\
\text { Support mother's desire to malntain the "normalcy" of } \\
\text { Michaed's teedings }\end{array}$ \\
\hline 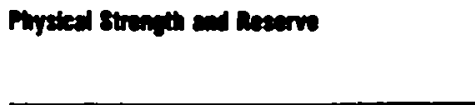 & $\begin{array}{l}\text { Promote parental control over Michael's AOC } \\
\text { Assist stati to recognize the benefits of parental } \\
\text { involvememt in Michael's physical care }\end{array}$ \\
\hline Entry & $\begin{array}{l}\text { Ensure adequate rest periods for mother } \\
\text { Ease power struggle between family and staff }\end{array}$ \\
\hline Knomberen & $\begin{array}{l}\text { Provide question and answer sessions } \\
\text { Clarify pros and cons of various feeding tochniques } \\
\text { Inform stafl of parent's neod for detailed, up-to-date } \\
\text { information }\end{array}$ \\
\hline Mnovatian & No deficit; continus to support \\
\hline 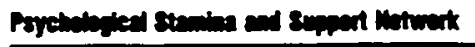 & No deficit; continue to support \\
\hline Dent syom & No deficit; continue to support \\
\hline
\end{tabular}

treatment plan.

Parental physical strength and reserve were fostered through interventions, which allowed the parents to maintain their control over the daily care of Michael whenever feasible during his hospitalization. Feeding, bathing, and comfort times were at their discretion. Soon the staff came to appreciate how much easier it was to care for Michael when his parents were there; his seizing and crying episodes decreased markedly. Energy was restored directly by ensuring adequate rest periods for Michael's mother and indirectly by easing the power struggle between his family and the staff.

Knowledge was promoted through frequent question and answer sessions with the $C$ family. Various feeding techniques were discussed and evaluated. The parents were encouraged to ask for clarification whenever necessary. The staff members in turn were able to appreciate the parent's legitimate need for up-to-date and detailed information as well as their need to participate fully in the decision-making process.

\section{Outcome}

Tension between the $C$ family and the staff did decrease over time, and a favorable solution to Michael's nutritional depletion was found. The nurs- ing staff began speaking of this family in more positive terms. Specific staff members commented on Michael's strikingly favorable response to his mother's presence in the room. In time the $C$ family came to trust the nursing staff. After the onset of Michael's second bout of pneumonia (precipitated by the surgery for GT tube placement) the $C$ family wanted to take him home immediately despite medical advice against this. However, the family did agree to delay discharge for an additional 24 hours on the recommendation of the nursing staff. The nursing staff in turn went to great lengths to coordinate the various aspects of Michael's homecare over those additional 24 hours so that $\mathrm{Mi}$ chael would not be discharged AMA. During a clinic follow-up visit two weeks later, Mrs. C voiced considerable satisfaction with the nursing care that Michael had received. In a nursing postdischarge conference on the $C$ family, the nursing staff applauded this family's ability to maintain control (i.e., nurturant power); they applauded as well their own actions in assisting this family to leave under circumstances acceptable to the entire health care team. Both staff and family benefited when the Family Power Resources model provided the framework for nursing interventions instead of using the more familiar crisis intervention framework.
In conclusion, not all families with a chronically ill, hospitalized child are in crisis. When crisis is not evident, the Family Power Resources model offers an attractive alternative to the crisis intervention framework. As this family case presentation illustrates, the model promotes the continuation of family self-care and determination throughout hospitalization so that disruption in current family functioning is minimized and the ease of subsequent home management is maximized. It is hoped that others will consider using the Family Power Resources model when assessing and evaluating the needs of a family with a chronically ill, hospitalized child.

\section{REFERENCES}

Adams, I. E., \& Lindemann, E. (1974). Coping with long-term disability. In Coelho, G. V., Hamburg. D. A., \& Adams, J. E. (Eds.), Coping and adaptation. New York: Basic Books.

Anderson, J. M. (1981). The social construction of ilt ness experience: Families with a chronically-ill child. loumal of Advanced Nursing, 6, 127-34

Kupst, M. J., Schulman, J. L., Honig, G., Maurer, H., Morgan, E., \& Fochman, D. (1982). Family coping with childhood leukemia: One year after diagnosis. Joumal of Pediatric Psycholosy, 7(2), 157-174.

Longo, D. C., \& Bond, L. (1984). Families of the handicapped child: Research and practice. Family Relations, 33, 57-65.

Mattsson, A., \& Gross, S. (1966). Social and behavioral studies on hemophilic children and their families. The Journal of Pediatrics, 68, 952-964.

Mckeever, P. T. (1981). Fathering the chronically ill child. Matemal Child Nursing, 6, 124-128.

Miller, J. F. (Ed.) (1983). Patient Power Resources. In J. F. Miller (Ed.), Coping with chronic illnes: Overcoming powerleseness. F. A. Davis, Philadelphia.

Pinyerd, B. J. (1983). Siblings of children with mylomenigocele: Examining their perceptions. MatemafChild Nurxins foumal, 12(1), 61-70.

Reif, L. (1975). Beyond medical intervention strategies for managing life in face of chronic illness. In Kramer, M. \& Strauss, A. (Eds.), Nursing in practice: A perspective on work environments. St. Lovis: C. V. Mosby, 1975

Solnit, A. J., \& Stark, M. H. (1961). Mourning and the birth of a defective child. Pyychoanabytic Study of the Child, 1b, 523-537.

Tropaver, A., Franz, M. N., \& Dilgard, V. W. (1970). Psychotogical aspects of the care of children with cystic fibrosis. American joumal of Diabled Chit dren, 119, 424-32.

Ventner, M. (1981). Familial coping with chronic and severe chilhood illness: The case of cystic fibrosis. Social Science and Medicine, 15A, 287.97.

Wrechter, E. U. (1977). Congenital anomalies. Nursing Forum, 16(3,4), 298-317.

White, R. W. (1974). Strategies of adaptation: An attempt at systematic description. In Coelho, G. V. Hamburg, D. A., \& Adams, J. E. (Eds.), Coping and adaptation. New York: Basic Books.

Williams, R. A. (1978). Crisis Intervention. In D. C. Longo \& R. A. Williams (Eds.), Clinical practice in poyctrosodal nursing: Asecument and intervention. New York: Appleton-Century-Crohs. 